\title{
MODELO DE PLANIFICACIÓN TERRITORIAL SOSTENIBLE DE LA MINERÍA: ENFOQUE EN AMÉRICA LATINA Y EL CARIBE
}

\author{
Adelso Nikolai Malavé Figueroa* \\ (iD) https://orcid.org/0000-0003-0479-1201 \\ Miguel Ysrrael Ramírez-Sánchez** \\ https://orcid.org/0000-0003-3045-5391 \\ Armando Anaya Hernández*** \\ https://orcid.org/0000-0003-3229-0930
}

RECIBIDO: Septiembre 2019 / ACEPTADO: Octubre 2019 / PUBLICADO: Enero 2020

\begin{abstract}
Como citar: Malavé Figueroa, Adelso; Ramírez-Sánchez, Miguel; Anaya Hernández, Armando. (2020).
Modelo de planificación territorial sostenible de la minería: enfoque en América Latina y el Caribe.

Telos: revista de Estudios Interdisciplinarios en Ciencias Sociales, 22 (1), Venezuela. (Pp.76-90).

DOI: www.doi.org/10.36390/telos221.06
\end{abstract}

\section{RESUMEN}

La investigación llevó por propósito estructurar un modelo para orientar a la planificación territorial, con base en la sostenibilidad, el quehacer en los espacios mineros, la gestión del riesgo de desastres en unión directa con la adaptación al cambio climático y la generación de resiliencia socio-ambiental en América Latina y el Caribe. Este fin se cimienta en los mandatos de todos los Objetivos para el Desarrollo Sostenible (ODS), Agenda 2030 (Naciones Unidas, 2019), las premisas de la Oficina de las Naciones Unidas para la Reducción del Riesgo de Desastres (UNISDR, 2017), las guías del Grupo Intergubernamental de Expertos sobre el Cambio Climático (IPCC, 2018) y, las recomendaciones del Consejo Internacional de Minería y Metales (ICMM, 2019). La propuesta presenta un diseño de campo no-experimental de corte transversal ajustado a un estudio de tipo proyectivo. Se aplicó un muestreo aleatorio simple con resultado de 67 ONG $(\mathrm{N}=80)$. El diagnóstico fue derivado a través de una escala con consistencia interna $(a=0,95 ; p<0,05)$. El Modelo Orientativo de la Planificación Territorial Sostenible de la Minería fue ensamblado siguiendo las asociaciones significativas entre sus componentes, los impactos socio-ambientales de la minería $(W=0,936 ; y=-0,867 ; p<0,01)$, y con respecto a la gestión de riesgo de desastres $(d=0,947 ; \gamma=1,000 ; p<0,01)$. El estudio

\footnotetext{
${ }^{*}$ Doctor(c) en Proyectos (UNINI-México). Magíster en Resolución de Conflictos y Mediación (Universidad de León-España), Licenciado en Sociología (UDO-Venezuela). Especialista en Investigación Holística y Análisis Estadístico. Consultor y voluntario internacional DD.HH. Correo electrónico: gestiondelriesgoamerica@gmail.com

** Doctor en Educación (UNINI), Maestro en Dirección Estratégica (Universidad de Belgrano), MBA (Universidad de Barcelona-España) y Licenciado en Informática (Instituto Tecnológico de Campeche-México). Coordinador del Doctorado en Proyectos en lengua portuguesa en la UNINI. Correo electrónico: miquel.ramirez@unini.edu.mx

*** Doctor en Arqueología (Universidad de Calgary), Arqueólogo y Profesor Asociado de las Universidades de Calgary (Canadá) y La Trobe (Australia). Colaborador en el Museo de Antropología de México y por el Instituto Nacional de Antropología e Historia de México Correo electrónico: armando.anaya@unini.edu.mx
} 
concluyó que el Modelo mitiga directamente las causas que gestan a los impactos socioambientales negativos y fomenta, mediante sus ejes transversales, la reducción de riesgos en los espacios mineros.

Palabras clave: planificación, gestión ambiental, adaptación al cambio climático, minería, resiliencia.

\title{
Sustainable territorial planning model of mining: focus on Latin America and the Caribbean
}

\begin{abstract}
The purpose of the research was to structure a model to guide territorial planning, based on sustainability, work in mining areas, disaster risk management in direct union with adaptation to climate change and socio-environmental resilience building in Latin America and the Caribbean. Such goal is based upon the policies of all the Sustainable Development Goals (SDGs), 2030 Agenda (United Nations, 2019), the premises of the United Nations Office for Disaster Risk Reduction (UNISDR, 2017), the criteria of the Intergovernmental Panel on Climate Change (IPCC, 2018), and the recommendations of the International Council on Mining and Metals (ICMM, 2019). The proposal presents a non-experimental cross-sectional field design adjusted to a projective study. A simple random sampling was applied with a result of $67 \mathrm{NGOs}(\mathrm{N}=80)$. The diagnosis was derived through a scale with internal consistency $(a=0.95 ; p<0.05)$. The Guidance Model for Sustainable Territorial Planning of Mining was assembled following the significant associations between its components, the socio-environmental impacts of mining $(W=0.936 ; \gamma=-0.867 ; p<0.01)$, and with respect to disaster risk management $(d=0.947 ; \gamma=1.000$; $p<0.01)$. The study concluded that the Model directly mitigates the causes that generate negative socio-environmental impacts and promotes, through its transversal axes, the reduction of risks in mining spaces.
\end{abstract}

Keywords: planning, environmental management, adaptation to climate change, mining, resilience.

\section{Introducción}

Hacia el último trimestre del año 2018, el Observatorio de Conflictos Mineros en América Latina (OCMAL, 2018), informó de la existencia de 254 conflictos socio-ambientales activos, 5 conflictos transfronterizos, 273 proyectos mineros directamente involucrados y 186 casos de criminalización de protestas comunitarias en contra de la minería. El panorama descrito denota una problemática profundamente enraizada en diversos espacios mineros de la región y también resulta ser acreedora de un carácter acumulativo y crónico. Por tal razón, resulta sustancial contar con mecanismos claros que permitan identificar y evaluar los riesgos como parte de los esquemas de planificación territorial, ya estableciéndose especialmente en el nivel local la relación entre los desastres, los efectos del cambio climático y la capacidad de afrontamiento socio-ambiental a estos eventos adversos. 
En torno a la incertidumbre apuntada en el parágrafo anterior, esta investigación quiso contribuir discerniendo acerca de los marcos referenciales, principios rectores, pautas, líneas estratégicas, pasos, acciones, resultados esperados y otros elementos primordiales que deben componer a un modelo orientativo sobre el proceso de Planificación Territorial Sostenible (PTS), especialmente dirigido hacia ámbitos locales y espacios mineros de América Latina y el Caribe. El presente trabajo se encuentra estructurado en tres partes: La primera consta de una contextualización teórica sobre la serie de riesgos principales usualmente implicados dentro de los espacios de actividad minera, así como también el complejo proceso de gestión de estos riesgos en combinación directa con los ajustes al calentamiento global, la resiliencia y la planificación del territorio bajo esquemas de sostenibilidad. La segunda parte aborda toda la metodología aplicada a la investigación (instrumentos, participantes y tipo de análisis de los datos). La tercera parte del estudio presenta resultados y conclusiones.

\section{Riesgos en los emplazamientos mineros}

De acuerdo con el International Council of Mining \& Metals (ICMM, Consejo Internacional de Minería y Metales, 2019), el concepto de minería se extiende por el conjunto de técnicas y actividades económicas del sector primario que lleva por fin la exploración, descubrimiento y extracción selectiva de yacimientos minerales, combustibles y otra serie de materiales. El Programa de las Naciones Unidas para el Medio Ambiente (PNUMA, 2004), especifica varios tipos potenciales de incidentes adversos que pueden presentarse en un emplazamiento minero, mayormente asociados a accidentes industriales y tecnológicos que pueden involucrar a ciertas acciones humanas negligentes y amenazas naturales combinadas. Entre estas acciones y amenazas podemos citar el uso inadecuado de recursos hídricos y de rutas de transporte, colmatación, movimientos telúricos, problemas de diseño y mantenimiento, equipamiento obsoleto, contención inapropiada de sustancias tóxicas, drenaje en deterioro y cimientos de planta industrial erosionados.

Un primer grupo de incidentes se reúne alrededor de los desperfectos en estructuras físicas como las presas y tuberías de relaves, botaderos de roca de desmonte, válvulas de lixiviación, depresión de taludes, entre otros. Un segundo grupo de incidentes incluye al vertido inapropiado de sustancias químicas y al manejo y transporte de materiales peligrosos. El tercer grupo de incidentes incorpora a las explosiones en planta, incendios, uso de explosivos (voladuras) y fuga de gases tóxicos hacia la atmósfera. Los tres grupos identificados en el parágrafo anterior implican serios impactos potenciales sobre la salud y los ecosistemas. Los riesgos van en gravedad ascendente desde la destrucción de la propiedad, pasando por la generación de nerviosismo y descontentos en la comunidad, daños serios a los sustratos naturales (flora y fauna), generación de enfermedades y lesiones, hasta la pérdida irreparable de vidas humanas.

\section{Gestión del Riesgo de Desastres (GRD) en la actividad minera}

Siguiendo a la Estrategia Internacional para la Reducción de Desastres (EIRD, 2009), la GRD es un proceso de múltiples acciones hacia el diseño y ejecución de políticas, planes, programas y cajas de herramientas rotatorias para estimular la prevención, minimización y control de los impactos de eventos adversos sobre las comunidades, ecosistemas, hábitats naturales y construidos. La GRD incluye sistemas integrales para evitar (prevención) o limitar (mitigación y preparación) los efectos destructores. Este proceso reclama la cooperación coordinada de 
gobiernos, sectores privados y voluntarios para que resulte. Este escenario deseable exige un importante y buen nivel de gobernanza sobre la administración de los recursos naturales, al igual que del personal que sea permanentemente capacitado en la evaluación de riesgos y en el diseño contextualizado de medidas socio-ambientales, últimas que deben ser coherentes con las características de cada territorio.

Los accidentes tecnológicos y las malas prácticas sobre los riesgos mineros no manejados señalan poderosas fallas y/o descuidos importantes en el diseño, ejecución, monitoreo y evaluación de los estándares operativos. Cuando una operación minera carece de los estándares de GRD o estos se incumplen, aparece por tanto una condición sub-estándar, lo cual supone una condición de riesgo intolerable dentro del espacio minero (Botín, 2019). La mencionada condición sub-estándar se apunta en calidad de vulnerabilidad con un radio de alcance variable en los territorios.

El riesgo industrial minero asciende en el instante en el que la probabilidad de ocurrencia de las amenazas sufre un repunte y la estimación de los impactos sobrepasa al riesgo aceptable. Los riesgos tecnológicos y aquellos relacionados con la negligencia en la ejecución de operaciones mineras pueden terminar en desastres, inclusive de mayores proporciones y extensión, debido a un efecto combinatorio con otros tipos de riesgos de orígenes natural y antrópico presentes y no atendidos sobre el territorio. En este punto se aprecia entonces una dinámica de variables con el rango y rigor necesarios para promover conflictos comunitarios, desequilibrios ambientales y el acuñado de movimientos de resistencia contra la minería por parte de los afectados (Toscana y Hernández, 2017).

\section{Adaptación al cambio climático (ACC), PTS y resiliencia}

La minería es un sector que aporta celeridad en el cambio climático por los cuantiosos consumos de energía, la contaminación y el uso indiscriminado de recursos naturales como el agua y la biomasa, aparte de las emisiones de gases de efecto invernadero (GEI) por las labores de extracción y beneficio. En sentido similar, la Comisión Económica para América Latina (CEPAL), ya alrededor del año 2002 calculaba que estas labores mineras constituían entre el 4 y $7 \%$ de la energía aprovechable (2010).

Frente al calentamiento global se emplaza la ACC como la capacidad compuesta por las sociedades en los niveles global, regional y local para ajustarse a las conversiones de la biósfera por los cambios producidos en el clima, asumiendo con criterio a los desafíos y nuevas oportunidades de tales alteraciones para aumentar la resiliencia (Casas, 2017). La GRD y la ACC poseen aristas comunes en sus ejercicios, tales como: reducir la incertidumbre ante las amenazas de tipo hídrico, meteorológico y biológico; acotar los niveles de fragilidad y fomentar la aparición de asentamientos humanos y ecosistemas más resilientes o dotados de una mayor capacidad de afrontamiento.

A propósito de la resiliencia, desde la perspectiva de las ciencias sociales se admite como la capacidad de un individuo, comunidad, sociedad y/o sistema activo para encarar, absorber, asimilar, sobrellevar, aprender, adaptarse y evolucionar de forma gradual ante la aparición de fenómenos contraproducentes que experimenta en su devenir (Cuvi, 2015). La resiliencia se gesta en las localidades por la vivencia y aprendizaje de los ciclos del desastre y las modalidades asumidas para recuperar la normalidad tras los impactos sufridos. 
Un enfoque deseable del desarrollo comparte la aspiración por cubrir los requerimientos ambientales y de las poblaciones mediante un abanico de políticas sostenibles, con el objetivo de orientar de forma eficiente los recursos del planeta sin comprometer, distorsionar ni colocar en serio riesgo la capacidad de las generaciones futuras para satisfacer sus propias necesidades (Bárcena y Prado, 2016; López, 2019; Severiche-Sierra et al., 2017).

El proceso de PTS puede contribuir con esta meta, al apuntarse como una herramienta de gestión del conjunto de patrimonios en el entorno físico-espacial y cultural, junto a la oferta de criterios para su ordenamiento y acciones basadas en la salvaguarda y uso racional de los recursos naturales, la reducción del riesgo de desastres, la estimulación de la equidad y calidad de vida y, además, garantías de bienestar en forma de nuevos ciclos de vida regidos por el progreso y la adaptabilidad ecológica (Zúñiga y Rodríguez, 2019).

\section{Metodología}

El trabajo de investigación comporta un tipo proyectivo, junto a un diseño de campo noexperimental, con corte transversal y un grado de estructuración cualitativo y cuantitativo. Fueron consideradas hipótesis descriptivas y de asociación entre las tres variables siguientes: la planificación territorial sostenible (PTS) en calidad de variable dependiente, mientras que los Impactos Socio-Ambientales de la Minerías (ISAM) y la Gestión del Riesgo de Desastres en convergencia teórica con la Adaptación al Cambio Climático y la resiliencia (GRDACC) fueron las variables independientes.

Se aplicó un muestreo probabilístico aleatorio simple, sin reposición y con criterio de saturación de datos, dando como resultado una muestra constituida por 67 Organizaciones NoGubernamentales (observatorios y similares en su mayoría), junto a un margen de error del $5 \%$ y una heterogeneidad del $50 \%$. Mediante uso del método holístico-hermenéutico (Rueda, 2017) en torno a las variables de la investigación se totalizaron 13 dimensiones, 29 sub-dimensiones y 69 indicadores para el instrumento.

El análisis global de los ISAM en una escala transformada de 60 puntos precisó cuatro rangos posibles de valoración mostrados en la Tabla 1.

Tabla 1. Valoración ordinal de los ISAM

\begin{tabular}{cc}
\hline Categoría & Rango \\
\hline Bajo & 1 a 15 puntos \\
Moderado & $>15$ a 30 puntos \\
Alto & $>30$ a 45 puntos \\
Severo & $>45$ a 60 puntos \\
\hline
\end{tabular}

Fuente: Elaboración propia.

El análisis global de la GRDACC y la PTS en una escala transformada de 60 puntos se indicó en cuatros rangos de posible valoración señalados en la Tabla 2. 
Tabla 2. Valoración ordinal de las escalas GRDACC y PTS

\begin{tabular}{cc}
\hline Categoría & Rango \\
\hline Excelente & $>45$ a 60 puntos \\
Alto & $>30$ a 45 puntos \\
Moderado & $>15$ a 30 puntos \\
Bajo & 1 a 15 puntos \\
\hline
\end{tabular}

Fuente: Elaboración propia.

El soporte para la definición conceptual y operativa de las variables se halla en referentes teóricos de Naciones Unidas (2019), el Intergovernmental Panel on Climate Change (IPCC, Grupo Intergubernamental de Expertos sobre el Cambio Climático, 2018), el International Council of Mining \& Metals (ICMM, Consejo Internacional de Minería y Metales, 2014a; 2014b), la Food and Agriculture Organization of the United Nations (FAO, Organización de las Naciones Unidas para la Alimentación y la Agricultura, 2017), la United Nations Office for Disaster Risk Reduction (UNISDR, Oficina de las Naciones Unidas para la Reducción del Riesgo de Desastres, 2017), y la Inter-Agency Standing Committee (IASC, Comité Permanente entre Organismos para la Asistencia Humanitaria, 2019).

El instrumento quedó representado como una encuesta sustentada en una escala de Likert, con preguntas cerradas de selección única por reactivo sobre la evaluación de la planificación territorial sostenible (EEPTS). La escala arrojó una excelente consistencia interna $(a=0,95 ; p<$ $0,05)$. Fueron realizados análisis descriptivos, correlativos, explicativos y predictivos de los datos para el diagnóstico; con base a pruebas estadísticas no-paramétricas tomando en cuenta el nivel de medición politómico-ordinal de las variables y la distribución de los datos que se ubicó fuera de la curva normal.

\section{Resultados}

\section{Características de la muestra}

La muestra estuvo conformada por hombres $(64 \%)$ y mujeres (36\%) en representación autorizada de sus ONG para los efectos de respuesta a la encuesta. El rango específico de edad registrado fue de 24 a 73 años con una media ubicada en el intervalo de 44 a 52 años de edad. El desempeño profesional de los ecologistas resultó el más frecuente $(11,94 \%)$, seguido por los profesionales del derecho $(10,45 \%)$, ingenieros ambientales $(8,96 \%)$, biólogos $(7,46 \%)$, sociólogos $(7,46 \%)$, antropólogos $(5,97 \%)$, comunicadores sociales $(5,97 \%)$ e ingenieros civiles $(4,48 \%)$. Otras profesiones reunieron el $37,31 \%$ entre los encuestados.

La procedencia de origen nacional de los participantes de la muestra fue $97,02 \%$ latinoamericana y caribeña, $1,49 \%$ especificó ser de origen canadiense y otro 1,49\% de origen español. Las nacionalidades con mayor frecuencia en la muestra fueron las siguientes: venezolana $(14,93 \%)$, colombiana $(8,96 \%)$, peruana $(7,46 \%)$, chilena $(7,46 \%)$, argentina $(7,46 \%)$ y mexicana $(5,97 \%)$. Los individuos encuestados de otras nacionalidades conglomeraron un $47,76 \%$.

En relación con el cargo u ocupación de los encuestados dentro de sus organizaciones, en el $20,90 \%$ de los casos se recibió respuesta del presidente o director principal, otro $20,90 \%$ 
perteneció al rango de coordinadores de algún programa o proyecto, 14,93\% indicó ser miembro activista o similar, $8,96 \%$ voluntarios y $34,31 \%$ se clasificaron en otras ocupaciones.

El área de trabajo y/o mayor desempeño de los consultados obtuvo una frecuencia superior en el tópico sobre industrias extractivas y minería, ya agrupando un $28,36 \%$ de los casos revisados. En segunda y tercera instancia se presentaron las áreas de medio ambiente y pueblos indígenas, reuniendo $19,40 \%$ y $16,42 \%$ de los casos, respectivamente. En cuarta y quinta posición se localizaron los sectores profesionales de derechos humanos con $10,45 \%$ y desarrollo sostenible con 8,96\%. Otras áreas de experticia profesional relacionadas con el cambio climático, resolución de conflictos, gestión de riesgo de desastres, participación comunitaria, protección civil, economía, planificación territorial, minería ilegal y crimen organizado abarcaron el 16,41\% restante.

\section{Evaluación descriptiva de la muestra con respecto a los puntajes globales de la EEPTS}

La observación del comportamiento de las variables permitió el cálculo del puntaje global del instrumento, es decir, la EEPTS con una nivelación transformada de 60 puntos, a través de la función descrita en la ecuación de propia autoría indicada a continuación:

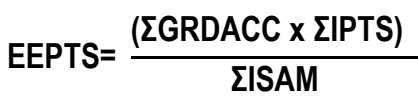

Donde,

EEPTS = Escala de Evaluación de la PTS en el marco de la minería con enfoque en la GRD, ACC y la resiliencia.

$\Sigma$ GRDACC = Sumatoria de los valores del Índice GRD asociados a la minería con enfoque en resiliencia y ACC.

$\Sigma$ IPTS = Sumatoria de los valores del Índice de Planificación Territorial Sostenible

$\Sigma I S A M=$ Sumatoria de los valores del Índice de impactos socio-ambientales asociados a las operaciones de la industria extractiva minera en América Latina y el Caribe.

El valor promedio de los ISAM registrado fue de 45,17 puntos (rango severo), el valor correspondiente para el GRDACC en esta oportunidad arrojó una media de 25,22 puntos (rango moderado) y el promedio obtenido para el IPTS fue de 25,2 puntos (rango moderado). Concatenado a estos resultados, el puntaje global de la EEPTS presentó una media de 16,44 puntos (rango moderado). La desviación típica fue de 11,65 y la varianza de 135,76.

En la Figura 1 se observa la distribución de las valoraciones en torno a la percepción de los encuestados en los cuatro rangos ordinales ya referidos, con predominio del grado bajo de la EEPTS en alrededor de $63 \%$ de los casos, seguido por los niveles moderado y alto, los cuales compartieron el segundo lugar al englobar el $36 \%$ de los casos y en tercer lugar el nivel excelente únicamente con $1 \%$ de las apreciaciones. 
Figura 1. Distribución de la valoración ordinal de la EEPTS.

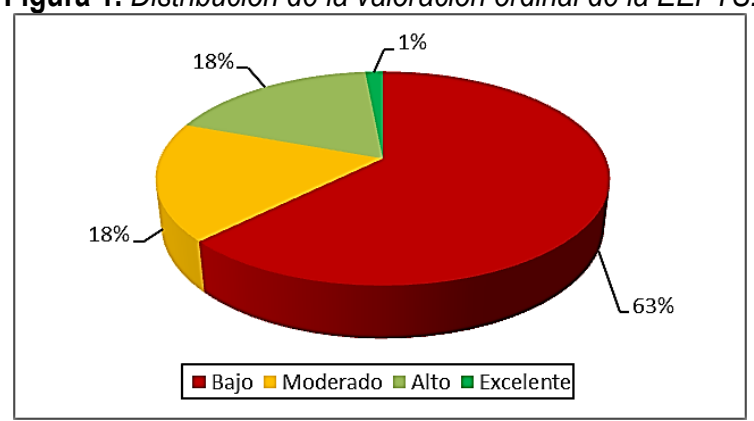

Fuente: Elaboración propia.

La media resultante como valor de la EEPTS entre los 44 casos considerados en Suramérica fue de 13,94 (rango bajo), resaltando el único caso atípico de Paraguay con una calificación alta (EEPTS $=36,02$ ) en una sub-distribución de datos con desviación típica de 7,77 y una varianza de 60,43. En los casos de México y Centroamérica, el valor promedio para la EEPTS en 18 de las percepciones se consolidó en 15,18 puntos (en un rango moderado), en una sub-distribución de datos con una desviación típica de 10,67 y una varianza de 113,95. El Caribe registra una EEPTS en rango bajo con una mediana de 9,13 puntos, además de Cuba como caso atípico ubicado en alta calificación (EEPTS $=38,39$ ), ya reportándose una desviación típica de 15,04 y una varianza de 226,16.

\section{Nivel de asociación entre las variables PTS, GRDACC e ISAM}

Fue ejecutado un Análisis de Componentes Principales para Datos Categóricos (ACPCat), el cual es un procedimiento construido y propuesto por el Data Theory Group de la Universidad de Leiden y por el Econometric Institute de la Universidad Erasmus Rotterdam, (Linting, Meulman, Groenen y Van der Kooj, 2007). Esta técnica sirvió en el nivel explicativo de este estudio para: 1) cuantificar de manera simultánea y óptima todas las variables ordinales de la EEPTS, 2) reducir el espectro dimensional de los datos con la mayor representación posible de las variables originales y, 3) establecer una base previa para la ponderación de las variables independientes en grado de relevancia sobre la determinación de la variable dependiente, con el fin de plantear pertinentes análisis predictivos.

A juzgar por los resultados, podemos indicar, preliminarmente, que los ISAM reservan una marcada asociación negativa con la PTS $(-0,570)$. Asimismo, la variable GRDACC ya muestra contribuciones asociativas elevadas con la PTS $(0,847)$. Al determinar el grado de concordancia entre los participantes (ONG) se aprecia que existe una asociación muy alta y significativa entre las variables $\left(W=0,936 ; X^{2}=125,444 ; p<0,01\right)$. En ese sentido, el valor del coeficiente de concordancia como medida de pronóstico nos permite aseverar en este estudio que los componentes de la planificación territorial sostenible de la minería en los ámbitos locales de América Latina están asociados, significativamente, con los impactos socio-ambientales de la actividad minera y los esquemas conceptuales de GRD enfocados en la resiliencia y la ACC.

Con el propósito de cualificar con mayor detalle la relación entre las variables y la dirección que sigue la relación de dependencia, fueron empleadas entonces medidas de asociación simétrica 
y direccionales. Comenzaremos por analizar la dirección de la dependencia entre los ISAM y la PTS, donde la segunda se acredita el mayor valor absoluto como variable dependiente en esta relación significativa con los ISAM, según el estadístico $d$ de Somers $(d=-0,516 ; p<0,01)$ con un error estándar asintótico reducido $(0,085)$.

El coeficiente Gamma, cuya oscilación de medida igualmente va de -1 a 1 , ya nos confirma la alta y significativa asociación entre aquellas variables $(y=-0,867 ; p<0,01)$, además de la existencia de un error estándar asintótico reducido $(0,082)$. La interpretación de estos estadísticos permitió aproximar en esta investigación que a mayor índice de impactos socioambientales negativos vinculados a las operaciones mineras, inferior será la simplicidad de diseño y ejecución de la planificación territorial sostenible de la minería en América Latina y el Caribe.

En otro orden, fue definida una clara dirección de dependencia de parte del índice de PTS respecto al índice de GRDACC, esto bajo condiciones de importante significación $(d=0,947 ; p$ $<0,01)$, además del reporte de un error estándar asintótico muy reducido $(0,03)$. El valor del coeficiente Gamma revalida esta información con un resultado de asociación simétrica, perfecta y significativa entre estas variables, en compañía de un error estándar asintótico nulo ( $y=1,000$; $p<0,01)$. La interpretación de estos resultados nos condujo a aceptar en este estudio que a mayor gestión de riesgo de desastres vinculados a la actividad extractiva minera, mayores serán las condiciones potenciales para el diseño, ejecución, monitoreo y evaluación de la planificación territorial sostenible de la minería.

\section{Análisis predictivo}

Para las predicciones o estimaciones puntuales se seleccionó el método de Análisis de Regresión Logística Categórica (REGCat), el cual facilita la realización de transformaciones no lineales y de esa manera analizar a las variables en diversos niveles, con el fin de ajustar el modelo predictivo a una ecuación de regresión lineal mediante el escalamiento óptimo ordinal (Navarro, Casas y González, 2010). Las variables predictoras mostraron contribuciones significativas a la variable respuesta, de forma positiva o negativa al tiempo aproximado de 24 meses de diseño y ejecución de planes territoriales sostenibles sobre la minería.

El grado de contribución positiva de la GRDACC en el aumento del éxito de la PTS fue más alto y significativo $(\beta=0,814 ; g l=1 ; p<0,01)$ que el nivel de contribución negativa de los ISAM en la reducción de éxito de la PTS $(\beta=-0,456 ; g l=2 ; p<0.01)$. El valor $F$ correspondiente a la distribución de la GRDACC ( $F=65,55)$ indica que su peso predictivo sobre la variable de PTS es comparativamente más consistente que el peso predictivo de los ISAM $(F=7,5)$.

Se estima una mejoría en la percepción de las ONG, por el tiempo estimado, acerca del proceso de planificación territorial sostenible en función directa de la intervención de la GRDACC y los ISAM. Sobre el particular, se predice en esta estimación que desaparecerán los registros en el rango bajo de la PTS (de un $18 \%$ original a un $0 \%$ pronosticado), el rango moderado incrementará 13 puntos porcentuales (de $63 \%$ original a un $76 \%$ pronosticado), el rango alto aumentará 3 puntos porcentuales (de $19 \%$ original a un $22 \%$ pronosticado) y el estimado para el rango excelente se adosará un $2 \%$ de los casos. 


\section{Descripción y justificación del Modelo Orientativo de la Planificación Territorial Sostenible de la Minería (MOPTSM)}

El MOPTSM es una propuesta sencilla de pautas propositivas y criterios para estimular el diseño, construcción, puesta en marcha, funcionamiento, monitoreo y evaluación de aquellos planes territoriales sostenibles que observen, con especial atención, el tratamiento de las labores mineras de extracción y beneficio en América Latina y el Caribe. El modelo se sincroniza con los productos concomitantes de la PTS de la minería focalizados desde los núcleos convergentes de la GRD y la ACC, lo cual se sustenta en los diagnósticos descritos y en la aspirada generación de asentamientos más resilientes.

Figura 2. Modelo MOPTSM: Sinopsis de actividades y expectativas.

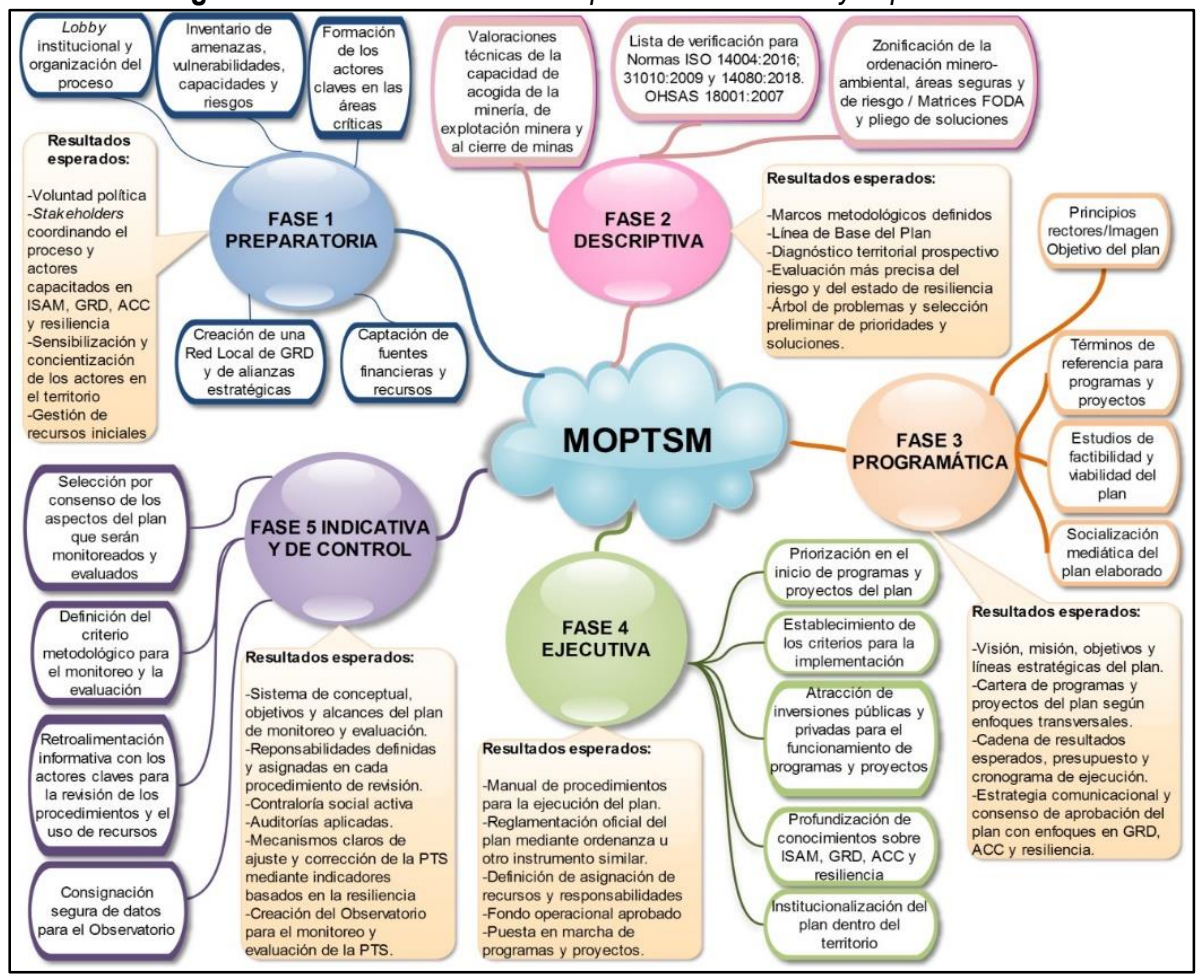

Fuente: Elaboración propia.

La propuesta espera, como escenario principal deseado, que la PTS de la minería identifique, afine y fortalezca claves de interpretación para la GRD, la ACC y la resiliencia como ejes transversales del desarrollo local; con el mismo rigor sobre las claves de interpretación en torno a la regulación local de la seguridad y de los ISAM, hasta niveles aceptables de riesgo por las 
comunidades y los ecosistemas. En la Figura 2, se observa una versión muy resumida del esquema general y los criterios de la propuesta MOPTSM.

\section{Objetivo del MOPTSM}

Proporcionar al sistema rector de la PTS minera de un boceto conceptual sucinto, propositivo y compuesto por criterios soportados en la triangulación entre la GRD, la ACC y la resiliencia socio-ambiental. Dicho sistema pretende integrar a las operaciones mineras bajo un esquema de seguridad que sea compatible con los ambientes natural y construido, con atención directa a los menesteres comunitarios y la gobernanza dentro del ámbito local.

\section{Claves de interpretación}

- La clave uno de interpretación, es decir, su punto de partida; lo constituye el marco institucional, legal y corporativo que rodea y aborda a todas las fases del proceso de planificación en cada territorio específico.

- La clave dos se refiere al patrón conceptual y operativo de las vulnerabilidades y amenazas tecnológicas presentes en los espacios mineros y sus formas de combinación temporal con otras amenazas de origen natural y antropogénico.

- La clave tres aduce directamente a los participantes y protagonistas que construyen el proceso, a su papel y manera singular de interactuar en cada territorio. La guía o modelo reúne en tres grandes grupos a la gama de actores participantes en el proceso en cuestión: 1) gobierno, 2) ciudadanía y, 3) sector de minerales (véase la Figura 3).

- La clave de interpretación número cuatro hace referencia al estilo de emprendimiento del proceso y las condiciones favorables para ello.

- La clave de interpretación número cinco, gira alrededor de los necesarios procedimientos de monitoreo y evaluación por intermedio de metodologías que garanticen también la contraloría social protagónica, permanente y evolutiva.

Figura 3. Actores centrales de la PTS minera.

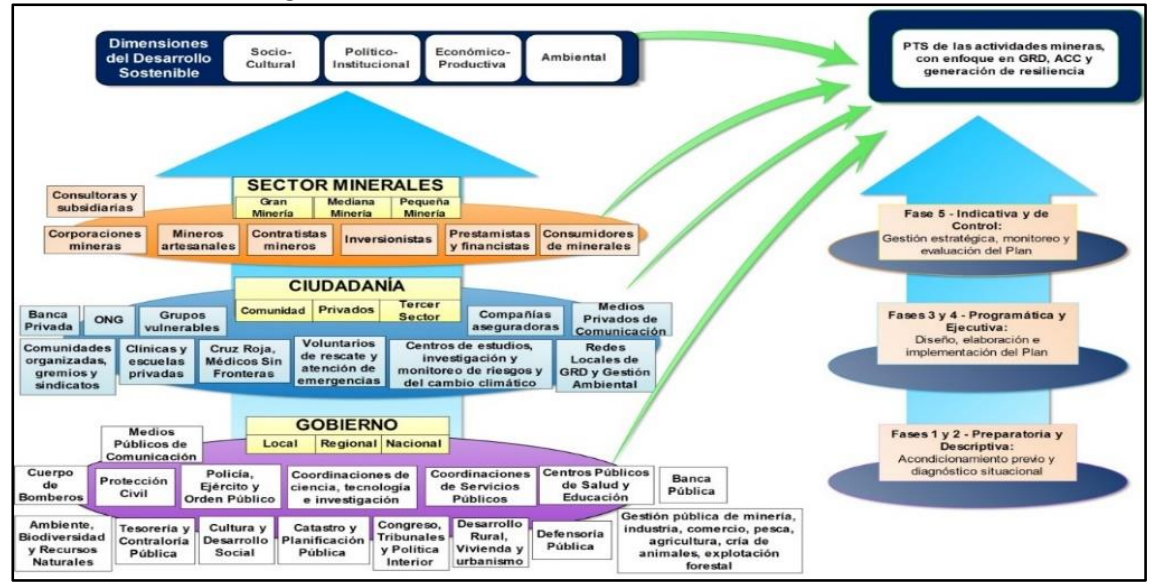

Fuente: Elaboración propia. 


\section{Derivaciones esperadas}

- Lineamientos claros y precisos entre los actores claves respecto a las vías de abordaje de su contexto histórico-concreto, realidad social y el nivel de desarrollo sostenible de su territorio.

- Disponibilidad efectiva de bases, criterios, métodos, estrategias y cajas de herramientas diversas para el diseño, ensamblaje y puesta en marcha de planes sostenibles concentrados en la actividad minera.

- Apoyo al ingenio entre los actores territoriales promotores del proceso, mediante instrucciones para los servicios de monitoreo y evaluación de los resultados, donde el ejercicio de contraloría estará acompañado por la capacidad para implementar ajustes programáticos, oportunos y apropiados hacia la sostenibilidad.

\section{Responsables y beneficiarios}

El modelo no predefine comitentes para evitar limitaciones, pero considerando que la PTS involucra a todos los actores de un territorio, ya sea que estos lo deseen o no; el comitente suele ser la máxima autoridad de gobierno en el ámbito local (presidente municipal, cámara de ediles, o sus equivalentes). Las instituciones y poderes públicos congregan la voluntad política para una indefectible iniciativa de este calibre. Una red local de GRD estaría comisionada y reconocida con cuota de responsabilidad en todas las supervisiones.

Directamente se anotan como beneficiarios a los entes gubernamentales y no-gubernamentales participantes en la organización de políticas, planes, programas y proyectos en torno a la gestión de los fenómenos adversos, vulnerabilidades y la ocurrencia de desastres. Gracias a la cooperación transparente con estas entidades se obtendrán las bases de datos necesarias para describir en detalle a la realidad minera local. Una gobernanza óptima o la intervención eficiente de las autoridades legítimas en cada territorio en efecto podrían, mediante uso diligente y supervisado de todos sus medios disponibles, intervenir con eficiencia para garantizar que los impactos positivos del modelo se distribuyan con mejor equidad y calidad.

Las empresas mineras y los actores de la pequeña minería también resultarían asistidos por esta propuesta, al ajustarse apropiadamente las zonas de seguridad interna y externa de los emplazamientos industriales y artesanales, también al combinar esfuerzos de GRD para la resiliencia comunitaria y los cuidados del hábitat. Conseguir estas metas tendría su reflejo en ganancias sobre la línea temporal, al evitar sanciones legales vinculadas con la amortización de daños por los impactos negativos de una minería con problemas de seguridad y de responsabilidad social. De forma indirecta se verían favorecidos las comunidades, ciudadanos y los usuarios de ecosistemas afectados y/o potencialmente perjudicados por desastres mineros, tales actores recibirían un compendio más oportuno de formaciones técnicas y de medidas tanto preventivas como compensatorias.

\section{Conclusiones}

La propuesta MOPTSM tiene unas pautas de interpretación para contextualizar con mayor éxito el ingreso de planes mineros en cada ámbito local, así como también el difundir los avances en informes de libre acceso con las mejores prácticas posibles en su cartera de proyectos, en cuyo caso se intenta responder a los cambios que desean percibir los usuarios, tomando en cuenta 
el intenso heteromorfismo territorial. La investigación realizada concluye en este sentido que a mayor GRD y ACC vinculados al quehacer minero, mayores posibilidades de triunfo podrá condensar la planificación territorial sostenible dentro de los ámbitos locales.

El modelo diseñado acude a la filosofía del empoderamiento social, participación inclusiva y protagónica, flexibilidad y heurística en las rectificaciones, transparencia en las revisiones y relaciones contables, promoción de la responsabilidad social corporativa, apropiación recursiva y sostenible de los medios para la vida, sensibilidad y prioridad de atención ante los sectores más vulnerables, el enfoque de género, la integración del estilo del Buen Vivir indígena, alocución de la sabiduría popular en toda la planificación, difusión veraz y democrática de finanzas y la adaptación a procesos globales como el cambio climático.

Con respecto al diagnóstico revisado para el modelo, podemos argumentar que existen claros indicios para una triangulación efectiva entre la GRD, la ACC y la resiliencia; por intermedio de su asociación significativa con el proceso de PTS en el ámbito local. En ese sentido, la GRD, la ACC y la generación de resiliencia pueden fraguar unidos varias dinámicas de acoplamiento en los espacios mineros, lo cual es factible cuando las instancias públicas, la ciudadanía y el sector de minerales se esmeran por combinar acciones comunes hacia el desarrollo sostenible.

Los actores al interactuar bajo aquel principio atesoran beneficios y legitiman al proceso de la PTS, lo que se traduce en la disponibilidad de instrumentos financieros eficaces y la transferencia democrática de conocimientos. De igual modo, las relaciones entre los participantes inspiradas en la confianza y la reciprocidad ofrecen una atractiva apertura para inversionistas, promotores, consultores y compañías de seguros que estén interesados en apostar a favor de la GRD, la ACC, el crecimiento de la resiliencia y por el auge de los dividendos en materia de la preservación de vidas, a partir de una minería que se deba no solo al lucro, sino también y, muy especialmente, al porvenir de la comunidad y de los ecosistemas.

Investigaciones posteriores y vinculantes pueden incorporar listas de verificación basadas en focos analíticos especíicos para nutrir las experiencias de PTS (género, derechos humanos, pobreza, empoderamiento social y otros), en virtud sintética de las aptitudes presentes en cada espacio minero. En el mediano y largo plazo la adición de nuevos enfoques integrales puede traducirse en un significativo aumento de los esfuerzos para el bienestar colectivo, el cuidado del ambiente natural y construido, la compenetración de los actores en sus relaciones cotidianas, la elevación del nivel en la gobernanza institucional, millones de dólares reservados en forma de ahorro por concepto de pérdidas controladas y la mejoría de la reputación de la industria minera con ejercicios fiscales transparentes.

En calidad de producto, el MOPTSM reúne las condiciones atractivas para que diversidad de actores puedan apostar por un proceso único en cada territorio, ya dotado de una lógica sostenible, preservación de vidas humanas y de ecosistemas, además de la alta rentabilidad económica que significa el ahorro millonario por la prevención de desastres mineros.

\section{Referencias bibliográficas}

Bárcena, Alicia., y Prado, Antonio. (2016). Agenda 2030 y los Objetivos de Desarrollo Sostenible. Una oportunidad para América Latina y el Caribe. Ediciones CEPAL. Chile. 
Botín, José. (2019). Los Desastres Mineros. ¿Cómo prevenirlos? Extraído de: http://www.emb.cl/construccion/articulo.mvc?xid=2556\&tip=11\&xit=los-desastresmineros-como-prevenirlos consulta: 22/07/19.

Casas, Marina. (2017). La transversalización del enfoque de género en las políticas públicas frente al cambio climático en América Latina. Ediciones CEPAL. Chile.

Comisión Económica para América Latina (CEPAL). (2010). El Desarrollo Sostenible en América Latina y el Caribe: Tendencias, avances y desafíos en materia de consumo y producción sostenibles, minería, transporte, productos químicos y gestión de residuos. Ediciones CEPAL. Chile.

Comité Permanente entre Organismos para la Asistencia Humanitaria (IASC). (2019). Índice de Gestión de Riesgos para América Latina y el Caribe. INFORM-LAC 2019. Extraído de http://www.inform-index.org/Subnational/LAC consulta: 22/07/19.

Consejo Internacional de Minería y Metales (ICMM). (2014a). Kit de Herramientas de Desarrollo Comunitario. Un conjunto de 20 herramientas revisadas y actualizadas para su aplicación en el ciclo de los proyectos de minería. Ediciones de ICMM. Reino Unido.

Consejo Internacional de Minería y Metales (ICMM). (2014b). Temas de liderazgo. Guía de gerenciamiento de riesgos fatales. Ediciones de ICMM. Reino Unido.

Consejo Internacional de Minería y Metales (ICMM). (2019) ¿Qué son los minerales y metales?

Extraído de: https://www.icmm.com/es/metals-and-minerals/producing-metals/queson-los-minerales-y-metales consulta: 03/08/19.

Cuvi, Nicolás. (2015). Un análisis de la resiliencia en Quito, 1980-2015. Bitácora Urbano

Territorial. Volumen 25, No 2. Colombia (Pp. 35-42). https://doi.org/10.15446/bitacora.v2n25.52036

Estrategia Internacional para la Reducción de Desastres (EIRD). (2009). Terminología sobre

Reducción del Riesgo de Desastres. Ediciones de UNISDR. Suiza.

Grupo Intergubernamental de Expertos sobre el Cambio Climático (IPCC) (2018). Global Warming of $1.5{ }^{\circ} \mathrm{C}$. Extraído de: https://report.ipcc.ch/sr15/pdf/sr15_spm_final.pdf consulta: $17 / 05 / 19$.

Linting, Mariëlle, Meulman, Jacqueline, Groenen, P., Van Der Kooj, Anita. (2007). Nonlinear principal components analysis: Introduction and application. Psychological Methods, Volumen 12, No 3. Estados Unidos (Pp. 336-358). http://dx.doi.org/10.1037/1082989X.12.3.336

López, Ricardo (2019). Bienestar y desarrollo: evolución de dos conceptos asociados al bien vivir. Telos. Volumen 21, № 2. Venezuela (Pp. 288-312).

Naciones Unidas (2019). Objetivos de Desarrollo Sostenible. Extraído de: http://www.un.org/sustainabledevelopment/es/ consulta: 25/06/19.

Navarro, Juan, Casas, Gladys, González, Emilio. (2010). Análisis de Componentes Principales y Análisis para Regresión para Datos Categóricos. Aplicación en la Hipertensión Arterial. Teoría y Aplicaciones, Volumen 17, No 2. Costa Rica (Pp. 199-230).

Observatorio de Conflictos Mineros de América Latina (OCMAL). (2018). Conflictos Mineros en América Latina: Extracción, Saqueo y Agresión. Estado de Situación en 2017. Ediciones de OCMAL. Ecuador. 
Oficina de las Naciones Unidas para la Reducción del Riesgo de Desastres (UNISDR). (2017). Plataforma Global 2017 para la Reducción del Riesgo de Desastres. Ediciones UNISDR. Suiza.

Organización de las Naciones Unidas para la Alimentación y la Agricultura (FAO). (2017). Lineamientos y recomendaciones para la implementación del Marco de Sendai para la Reducción del Riesgo de Desastres en el Sector Agrícola y Seguridad Alimentaria y Nutricional América Latina y el Caribe. Ediciones de FAO. Italia.

Programa de las Naciones Unidas para el Medio Ambiente (PNUMA). (2004). APELL para minería. Guía para la industria minera a fin de promover la concientización y preparación para emergencias a nivel local. CETEM/CYTED. Brasil.

Rueda, Diana (2017). Enfoque de la Hermenéutica a través de sus elementos conceptuales. Analéctica, Volumen 24, No 3. Argentina (Pp. 1-4).

Severiche-Sierra, Carlos, Bedoya-Marrugo, Elías, Meza-Alemán, María y Sierra-Calderón, Darío (2017). Gestión para la Sostenibilidad Ambiental, Socio-cultural y Económica en el Sector Hotelero: Revisión de la Literatura. Telos, Volumen 19, No 2. Venezuela. (Pp. 475-495).

Toscana, Alejandra y Hernández, Pedro. (2017). Gestión de riesgos y desastres socioambientales. El caso de la mina Buenavista del cobre de Cananea. Investigaciones geográficas, Volumen 93. México (Pp. 1-14). http://dx.doi.org/10.14350/rig.54770

Zúñiga, Libys Martha y Rodríguez, Jorge Luis. (2019). Experiencias del Plan de Ordenamiento Territorial. Mayarí, Cuba. Bitácora Urbano Territorial, Volumen 29, № 2. Colombia (Pp. 135-142). https://doi.org/10.15446/bitacora.v29n2.63010 